\title{
Visual Indication while Sharing Items from a Private 3D Portal Room UI to Public Virtual Environments
}

\author{
Minna Pakanen ${ }^{1}$, Leena Arhippainen ${ }^{1}$, \\ Jukka H. Vatjus-Anttila ${ }^{1}$, and Olli-Pekka Pakanen ${ }^{2}$ \\ ${ }^{1}$ Intel and Nokia Joint Innovation Center, Center for Internet Excellence, \\ P.O. Box 1001, FI-90014 University of Oulu, Finland \\ ${ }^{2}$ University of Oulu, Department of Information Processing Science, \\ P.O. Box 3000, FI-90014 University of Oulu, Finland \\ \{minna.pakanen, leena.arhippainen, jukka.vatjus-anttila\}@cie.fi, \\ opakanen@paju.oulu.fi
}

\begin{abstract}
In this paper, we describe the user experience evaluation results of a 3D Portal Room UI for sharing 3D objects from private space to remote public virtual environments. The user evaluation with 30 participants was conducted with a functional prototype and additional high quality images that were printed on paper sheets. The evaluation indicated that participants liked this way of sharing objects and found it also useful. However, it also raised some privacy concerns, especially if the target virtual environment was perceived as public. Evaluation elicited that the visual indication while sharing objects is important; therefore, designers of 3D virtual environments should prefer a distinguishable glow around the shared object and portal.
\end{abstract}

Keywords: Portals, 3D user interface, virtual environment, user interaction, visual indication, user experience.

\section{Introduction}

In collaborative three dimensional (3D) virtual environments (VEs), such as Second Life [9] and World of Warcraft [2], users can see objects and other people's avatars in a 3D space. To share items with other users or players, current solutions must rely on two dimensional (2D) pop-up repository windows, which reduce the view of the VE and weaken the $3 \mathrm{D}$ experience. There is a need for a private $3 \mathrm{D} \mathrm{VE}$ which is located in parallel with public VEs and the possibility to move objects between them. The need is noted in collaborative VE context, where a user wants to have private information in collaborative VE, but at the same time, he/she needs to be able to hide it form other users [3]. There is also a need in education context, where a teacher could prepare classes and share material from his/her private 3D space to the pupils in public virtual classrooms, and at the same time, be able to monitor what pupils are doing in collaborative VEs [1]. 
In this paper, we present a 3D Portal Room UI with an ability to have four client server connections in parallel, which are presented to the users as four parallel views to the VEs. The focus of this paper is on visual indication when moving 3D objects from a private 3D Portal Room UI to public VEs. We report the user evaluation of the prototype and additional visual indication image examples with 30 participants. This paper contributes to the 3D VE research and the designer community by presenting user experiences (UXs) [4] with the prototype and participants preferences on visual indication while objects are shared through portals.

\section{Related Research}

Portals have been studied in prior research from the navigation point of view [7]. Kotziampasis et al. [7] found that portals help users to navigate in the virtual worlds, because of the visual presentation of the target space. Portals have also been used as object transfer devices, but only in two dimensional environments, where documents and 2D shapes can be exchanged through portals on a surface display [15].

The limitation of prior research is the lack of user interaction research. Shneiderman [14] argued ten years ago about making 3D UIs to facilitate user tasks with an enhanced 3D design rather than just mimicking reality in all possible ways. To design visually enhanced 3D UIs and games, we agree with El-Nasr and Yan that visual indication is an important factor, especially for novice users [5].

Visual attention process has been studied extensively within cognitive science and psychology, but not from a 3D game perspective [5]. Visual indication in 3D scenes has been studied from the anchor highlighting point of view, where the colour coding on 3D objects was preferred in the usability test and user comments, but the most visually appealing choice was the colour edges [11]. Also, it is noted in 3D games research that the location of the visual indication is as important as the visual indication itself [5].

Butz et al. [3] have studied indication of shared elements in collaborative 3D VEs from privacy perspectives. They introduced two methods for privacy management: a vampire mirror, which shows only shared items, and a privacy lamp, in which a beam of light depicts the private items of other objects in collaborative 3D VE in augmented reality situation [3]. Also, it is suggested in prior research that the colour of the shared element should be changed or other visual feedback be given when something is moved from a personal 3D GUI to the collaborative 3D VE [10].

\section{The Evaluation Prototype}

We used the realXtend Tundra 2 [12] virtual world viewer as a setup platform for portals. It is based on a client-server type architecture. We used the following hardware setup in the user evaluation: HP Elitebook 2760p laptop with Ubuntu Linux 12.04 LTS 32bit with WLAN 802.11g public Internet connection. In addition, we had an external 24" Dell P2411Hb 1920x1080 monitor, mouse and keyboard. 
The 3D Portal Room UI acts as a private 'log-in screen' to the other VEs. Portals provide an ability to have four client-server connections in parallel, which are presented to the users as four parallel views to the VEs [6]. We decided to use a room metaphor, because it creates a feeling of privacy [10]. As we wanted to increase the feeling of private space, there are no windows in the room and the wall texture was chosen to look sturdy (Fig.1). In the 3D Portal Room UI, there are the user's personal items such as a microphone, a photo album, a notebook, a trashcan, a game and MS PowerPoint, Word, PDF files. The portals (views) to the public VEs are covered with round doors, when there is no open connection to them. Connection is created by tapping the door, when a door mesh is replaced by a real-time view to the remote VE. We chose four remote public VEs (Music Club, Office, Outdoor Music Club and City) for the prototype. The chosen VEs had different visual styles from each other, because we wanted to study how participants perceive sharing items to them. (Fig. 1.)

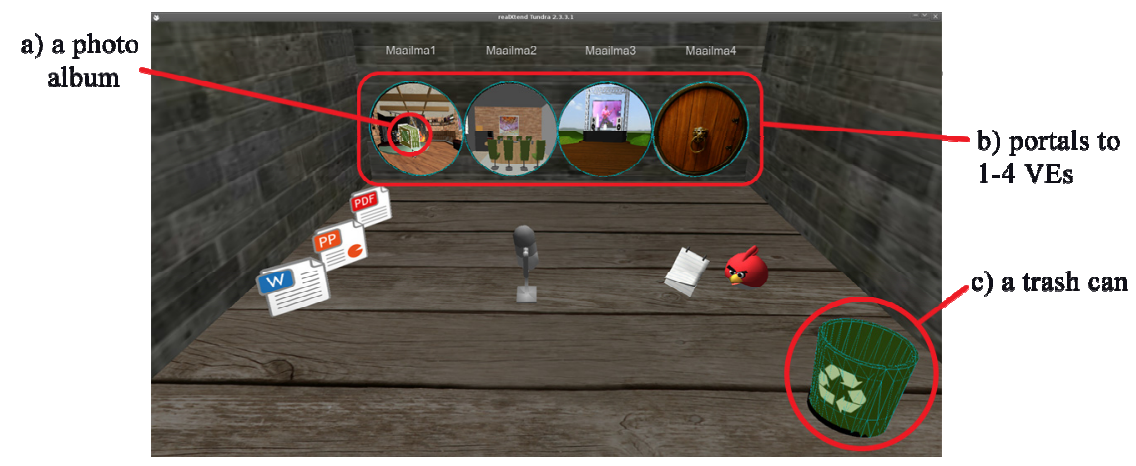

Fig. 1. In the 3D Portal Room UI, the visual indication is showed on the selected object (a) and on possible targets $(b, c)$ when a user is sharing a photo album to the Music Club VE

We implemented to the 3D Portal Room UI a functionality to move private 3D objects to remote VEs and destroy objects from the private UI by moving them to a $3 \mathrm{D}$ trashcan. The visual indication for the selected object and targets was implemented with turquoise lines and green overlay colour on the object's mesh (Fig. 1).

The idea was to implement the prototype on a touch screen device, but due to limitations of the Linux Ubuntu operating system, it was not a suitable solution. Therefore, we decided to evaluate the prototype with mouse input to get user feedback on the portal idea of moving items through portals and the visual indication while doing it. The main idea with implemented interactions was that a user selects an object by pressing the left mouse button, and then he/she moves the cursor on the VE portal, when an object 'jumps' on the portal. The user then presses the left mouse button and a copy of the object drops on the ground into the VE and the original object returns to its place in the 3D Portal Room UI. We also implemented realistic physics for the shared object when it drops into the VE. Thus, we did not define the position where the shared object should land in the VE; therefore, the objects landed with a random place and orientation. 


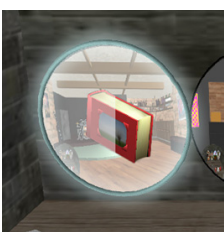

A

White dimming on the portal White dimming on $1 / 2$ of the icon Colour on the portal edge

Glow effect on the portal edge $3 \mathrm{D}$ effect on the portal edge

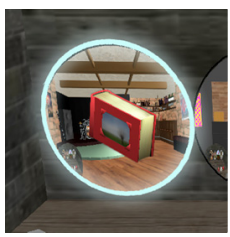

E

Colour on the portal edge Glow effect on the portal edge

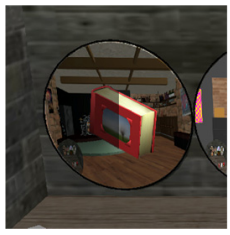

H

Black dimming on the portal Black dimming on $1 / 2$ of the icon

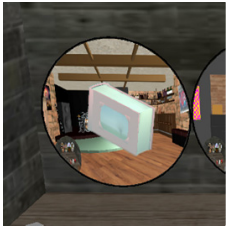

L

Turquoise dimming on the icon

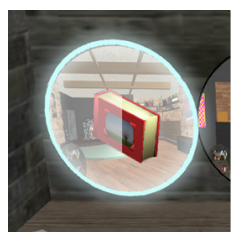

B

White dimming on the portal White dimming on $1 / 2$ of the icon Colour on the portal edge Glow effect on the portal edge

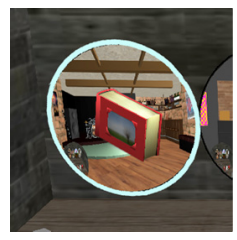

F

Colour on the portal edge

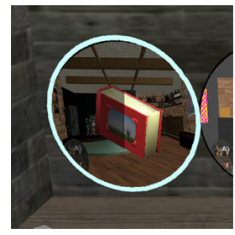

I

Black dimming on the portal Black dimming on $1 / 2$ of the icon Colour on the portal edge

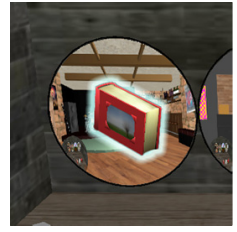

M

Glow effect on the icons' edge (turquoise)

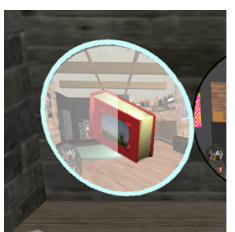

C

White dimming on the portal White dimming on $1 / 2$ of the icon Colour on the portal edge

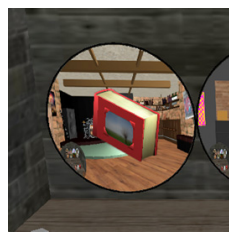

G

No effects

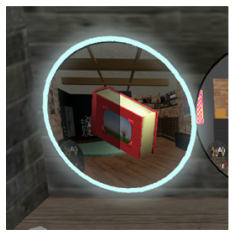

J

Black dimming on the portal Black dimming on $1 / 2$ of the icon Colour on the portal edge Glow effect on the portal edge

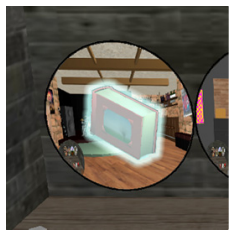

N

Turquoise dimming on the icon Glow effect on the icons' edge

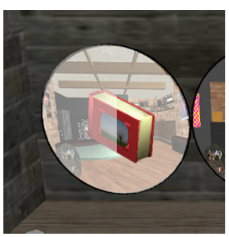

D

White dimming on the portal White dimming on $1 / 2$ of the icon

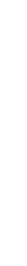

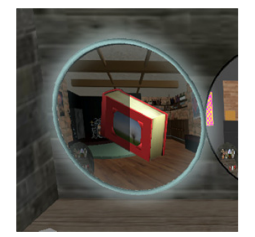

K
Black dimming on the portal Black dimming on $1 / 2$ of the icon Colour on the portal edge Glow effect on the portal edge $3 \mathrm{D}$ effect on the portal edge

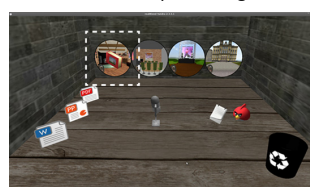

Original example $\mathbf{G}$ Size: $28,5 \times 16 \mathrm{~cm}$

Fig. 2. Enlargement of the visual indication effects used in the example images (A-N). Below an example of the original image with the enlargement area indicated with dashed white line

\section{Example Images for Evaluation}

To study what kind of visual indication users would prefer for indicating moving objects from their private 3D UI area to public VE, we prepared fourteen different kinds of indications either on the object or on the target portal (Fig. 2). We used as an inspiration object highlights that we had noticed in games, such as Serious Sam 3 BFE [13]. We wanted to extend the visual indications used in prior research [11] and 
investigate suggested solutions [10]; therefore, we made several versions with different mixtures of colour overlay, colour edges and glow. We decided to use a uniform colour theme in all options, to make them easily comparable with each other. Indications were made in the Adobe Photoshop by adding different kinds of layer styles for the screenshot image of the 3D Portal Room UI. We decided to present visual indication examples as images printed on $28,5 \times 16 \mathrm{~cm}$ sized paper, because then participants can compare examples easier by taking them into their hands. It is also a good way for collecting user experiences in an early development phase and it is also more costeffective and faster than implementing all possible solutions to the prototype [10].

\section{$5 \quad$ User Evaluation}

We recruited 30 participants for the user evaluation. The participants' age varied from 20 to 52 years with a mean of $32.66 \%$ were male. $60 \%$ of the participants used 3D VEs on a computer, but none on a tablet or a mobile phone.

A user evaluation had two parts. In the first part, participants familiarized themselves with the prototype by doing simple tasks with it. In the second part, subjects ranked three best options from given examples (A-N) to represent visual indication while an object is moved from the private 3D UI to a public VE. Subjects were interviewed and their subjective experiences were gathered by observing, writing down their comments and video recording while completing the following tasks:

1. Open connections to the VEs (click doors) \& explain if the VE is public or private.

2. Share objects from the private 3D Portal Room UI to public virtual environments:

(a) Share Power Point slides to the Office scene (drag PP icon to VE 2)

(b) Give feedback to the City council (City scene) (drag W icon to VE 4)

(c) Delete an old PDF file (drag PDF icon to the trashcan in 3D Portal Room UI)

(d) Share a photo album to the Music Club (drag photo album 3D icon to VE 1).

Comment on the following question: Do you find sharing (1) easy, (2) useful, (3) secure, and (4) what kind of indication there should be presented while sharing.

3. Select from given alternatives (A-N) the best, 2nd best and 3rd best option for indicating an object moving through the portal to a public VE. The images were placed on the table in random order for each subject. Participants were asked to mark their choice with little Post-it notes and comment each selection.

\section{$6 \quad$ Findings}

For all of the participants, it was easy to understand the logic that by clicking the door a connection opens to the VE. When sharing the objects, $70 \%$ of the users interacted directly as intended and $27 \%$ did it correctly in the second try. $3 \%$ did it right in the third try. The main problem with the interaction during sharing was that participants did not press the left mouse button on the portal, to drop a copy of the object to the VE. As one user commented: "Why I have to press (left button) -it is weird... I would just drag it there". Also the object transition implemented in the prototype caused 
confusion for a few participants when they were sharing objects. As they moved the cursor slowly, it seemed that the selected object did not follow the cursor at all. As a consequence, users thought that they have not selected the object at all. As one user stated: "(object) should follow (the cursor) smoothly and not just jump on the portal".

\subsection{Security Concerns while Sharing Objects}

In general, participants liked the possibility to share items to the remote VEs and they also found it useful, especially with the case of sharing a PowerPoint file to the Office. However, sharing also raised privacy and security concerns, especially with VEs that participants thought to be open for all people. All of the participants thought that outdoor VEs (Outdoor Music Club and 3D City) were public. Also the indoor Music Club was perceived to be public by $97 \%$ of the participants. The Office was perceived public only by $57 \%$ of the users. Participants explained that they perceived it either private or semi-private because the environment was quite small and the amount of the chairs in the scene created a feeling that it is meant for a limited amount of attendees. Subjects explained also that in real-life, meeting rooms are meant for private gatherings.

\subsection{User Experiences with the Visual Indication in the Prototype}

Participants liked the realistic physics when a shared object was dropped to the virtual environment. It was found as important but also amusing. They commented it as: "Wow", "Funny" and "visually attractive". One participant commented: "Dropping is a good effect indicating that (an object) goes to the virtual environment". Participants did not like the fact that the objects did not land in a rational location in the scene and they wished to be able to organize and move the objects in the VE after sharing them.

The implemented indication while sharing was not perceived to be visible enough. Participants commented that they wished to have clearer visual feedback on the edge of the shared item or/and on the portal, such as "light", "flash of light" or "colour change". Also audio feedback was recommended. What is more, a few wished for a check-up window with text: "do you want to share this item to the ..." to pop-up.

\subsection{User Experiences with the Visual Indication Example Images}

The distribution of participants' preferred visual indications is presented in Figure 3. To weight the primary choices, we used Borda count [8]. We gave three points for participants' 1st choice, two points for their 2nd choice, one point to the 3rd choice and zero points to all other options.

A non-parametric Kruskall-Wallis test indicated significant differences between the data points for the fourteen different alternatives $(\chi 2(13)=27,530, p<0,02)$. In the preference ranking task, participants preferred option $\mathrm{M}$ (turquoise glow effect around the edge of the photo album) for indicating objects transferring from private 3D UI to the public VE. It received the highest amount in the Borda count (36) and $27 \%$ of the participants rated it as the best choice. Participants explained that the 


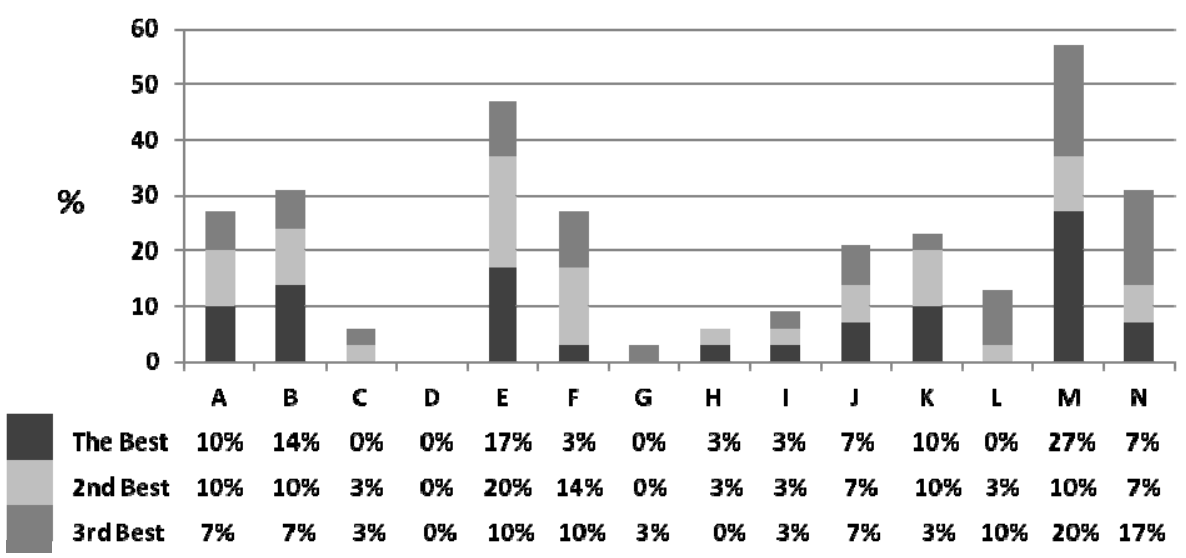

Fig. 3. Preference ranking results for the visual indication

indication of the object is the most important thing to be represented. They also commented that when the visual indication is on the object it looks like something is happening; the object is moving to the other VE. The glow effect was also perceived as "visually pleasant", "easily noticeable" and "it really makes the object to pop-up".

The second highest was option E (turquoise colour + glow effect around the edge of the portal). It received 30 points and $17 \%$ of the first-place votes. Participants explained that the most important thing is now highlighted (e.g. the target). As one participant commented: "I have already chosen the object, so the glow indicates where to drop it". It was also suggested that both the object and the portal could glow.

The third highest was option B (white dimming on the portal view and on half of the photo album, turquoise colour + glow effect around the edge of the portal) with 20 points and $14 \%$ of the first-place votes. It was preferred because it gave an impression that the object is already half way through to the other VE, therefore, it looked intuitive. Also the indication on the portal was large enough to be noticed, as one participant commented: "(the indication should be) as visible as possible to preventing unintentional sharing to the other virtual environment". Despite different user views, a pairwise comparison between the three most popular alternatives revealed no significant statistical difference between them.

\section{Conclusion}

In this paper, we present a private 3D Portal Room UI for sharing 3D objects to public VEs. We present user evaluation with 30 participants and results on both; interaction with a functional prototype and a preference ranking of high quality example images. The participants found the sharing of objects from a private 3D UI space to public VEs as easy and useful, especially when sharing a PowerPoint file to the Office scene. The visual indication should be clear when an object is moved out of the private UI area. The evaluation indicated that designers of 3D VEs should prefer a distinguishable glow effect around the shared object and the target portal. 
Whereas we believe that our results provide useful information for researchers and designers of the 3D VEs, we also recognize the limitations of the study. As we decided to use printed paper sheets as examples in the preference ranking task, we missed the interaction with the examples. However, the method provided us a lot of UX based information in the early development phase and was cost-effective and time-saving. With the feedback, we now know how to proceed with the design and implementation of portals. In the future, we are going to study how the visual indication works with the touch interaction. It would also be interesting to study how shared objects should be indicated to the users of public VEs.

\section{References}

1. Arhippainen, L., Pakanen, M., Hickey, S., Mattila, P.: User Experiences of 3D Virtual Learning Environment. In: 15th International Academic MindTrek Conference, pp. 222227. ACM Press, New York (2011)

2. Blizzard Entertainment Inc. World of Warcraft, http: / / us . battle.net/wow/en/

3. Butz, A., Beshers, C., Feiner, S.: Of Vampire Mirrors and Privacy Lamps: Privacy Management in Multi-User Augmented Environments. In: 11th Annual Symposium on User Interface Software and Technology, pp. 171-172. ACM Press, New York (1998)

4. ISO DIS 9241-210:2010. Ergonomics of human system interaction - Part 210: Humancentred design for interactive systems. International Standardization Organization (ISO). Switzerland

5. El-Nasr, M.S., Yan, S.: Visual Attention in 3D Video Games. In: Advances in Computer Entertainment Technology, Article No. 22. ACM Press, New York (2006)

6. Hickey, S., Arhippainen, L., Vatjus-Anttila, J.H., Pakanen, M.: User Experience Study of Concurrent Virtual Environments with 2D Tab and 3D Portal UIs. In: 19th International Conference on Engineering, Technology and Innovation, ICE, June 24-26 (2013)

7. Kotziampasis, I., Sidwell, N., Chalmers, A.: Seamlessly Integrated Distributed Shared Virtual Environments. In: 20th Spring Conference on Computer Graphics, pp. 138-147. ACM Press, New York (2004)

8. The mathematics of voting: Democratic symmetry. The Economist, p. 83 (March 2000)

9. Linden Lab. Second Life, http: / / secondlife.com/

10. Pakanen, M., Arhippainen, L., Hickey, S.: Studying Four 3D GUI Metaphors in Virtual Environment in Tablet Context. In: 6th International Conference on Advances in Computer-Human Interactions, pp. 41-46. ThinkMind Press (2013)

11. Pichler, M.: Interactive Browsing of 3D Scenes in Hypermedia: The Hyper-G 3D Viewer, http://ftp.iicm.tugraz.at/pub/theses/mpichler.pdf

12. realXtend, http://realxtend.org/

13. Serious Sam 3 BFE, http: / / store. steampowered. com/app/41070/

14. Shneiderman, B.: Why Not Make Interfaces Better than 3D Reality? IEEE Computer Graphics and Applications 23(6), 12-15 (2003)

15. Voelker, S., Weiss, M., Wacharamanotham, C., Borchers, J.: Dynamic Portals: a Lightweight Metaphor for Fast Object Transfer on Interactive Surfaces. In: ACM International Conference on Interactive Tabletops and Surfaces, pp. 158-161. ACM Press, New York (2011) 